\title{
Rapid Enlargement of Vitiligo Vulgaris after Initiation of Dupilumab for Atopic Dermatitis: A Case Report
}

Shintaro TAKEOKA ${ }^{1,2}$, Masahiro KAMATA ${ }^{2 *}$, Ikumi YOKOI ${ }^{1}$, Aya TAKEHARA ${ }^{1}$ and Yayoi TADA ${ }^{2}$

${ }^{1}$ Takeoka Dermatology Clinic, Kagawa, and 'Department of Dermatology, Teikyo University School of Medicine, 2-11-1 Kaga, Itabashi-ku, Tokyo, 173-8605, Japan.*E-mail: mkamata-tky@umin.ac.jp Accepted Oct 25, 2021; Epub ahead of print Oct 25, 2021

Dupilumab, a monoclonal antibody targeting interleukin-4 receptor $\alpha$, demonstrated high efficacy and effectiveness for atopic dermatitis (AD) with tolerable safety in clinical trials (1) and real-world data $(2,3)$. Vitiligo vulgaris is an acquired leukoderma characterized by depigmentation of the epidermis resulting from destruction of melanocytes. We report here a patient with $\mathrm{AD}$ with a small incomplete patch of depigmentation of vitiligo vulgaris on his forehead, which enlarged after initiation of dupilumab.

\section{CASE REPORT}

A 17-year-old male with severe AD presented to our clinic with refractory nodules accompanied by severe pruritus spreading over his entire body (Fig. 1A). He had received topical corticosteroids, oral antihistamine, and phototherapy for severe AD since he was diagnosed with $\mathrm{AD}$ at the age of 2 years. He initiated dupilumab when it became available for the treatment of $\mathrm{AD}$ in Japan. When dupilumab treatment was started, his Eczema Area and Severity Index (EASI) was 44.5, af-
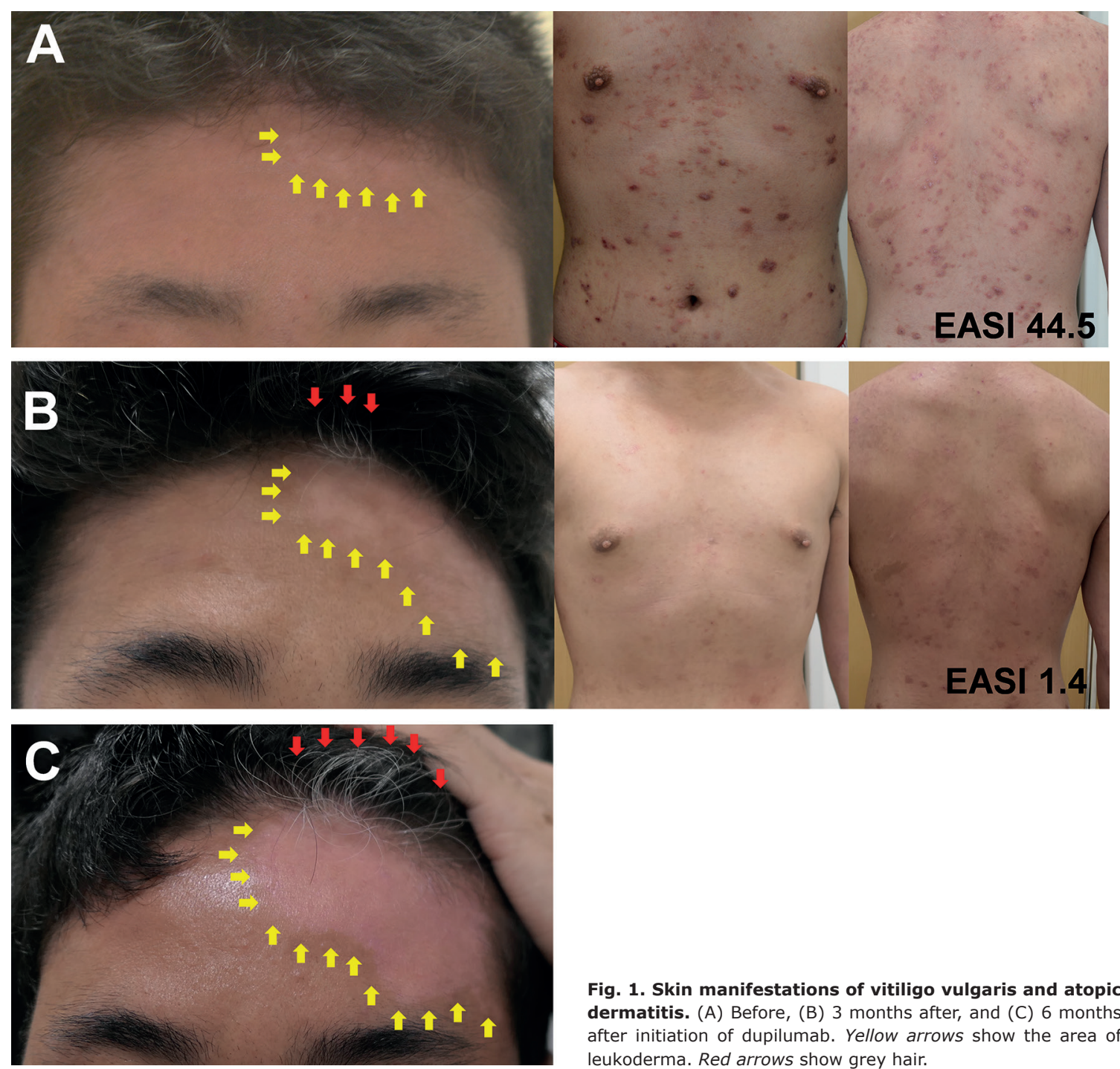

Fig. 1. Skin manifestations of vitiligo vulgaris and atopic dermatitis. (A) Before, (B) 3 months after, and (C) 6 months after initiation of dupilumab. Yellow arrows show the area of leukoderma. Red arrows show grey hair. 
fected body surface area (BSA) 75\%, and Investigator Global Assessment (IGA) score 4. The serum levels of thymus and activation-regulated chemokine (TARC) and IgE were $1,980 \mathrm{pg} / \mathrm{ml}$ and 9,648 IU/ml, respectively. Photographs taken at the time of initiation of dupilumab showed a patch of incomplete depigmentation on his forehead (Fig. 1A); however, because the leukoderma was slight and small, neither he nor the physician noticed it at that time. Although dupilumab significantly improved skin manifestations of $\mathrm{AD}$, including nodules and pruritus, as shown in Fig. 1B (EASI 1.4, affected BSA 4\%, IGA 1, serum TARC level $271 \mathrm{pg} / \mathrm{ml}$, and serum $\mathrm{IgE}$ level $3,051 \mathrm{IU} / \mathrm{ml}$ at 3 months after initiation of dupilumab), the leukoderma enlarged rapidly, was accompanied by grey hair, and became clinically conspicuous (Fig. 1B, C). He was treated with hydrocortisone butyrate ointment, and, subsequently, delgocitinib ointment, in addition to 10 sessions of excimer light therapy for the patch of complete depigmentation, but it did not ameliorate vitiligo vulgaris. He withdrew from dupilumab treatment 13 months after the initiation of dupilumab due to economic reasons. To date, 17 months after the cessation of dupilumab, slight repigmentation has been observed, but its area has not shrunk.

\section{DISCUSSION}

In this patient, although dupilumab rapidly improved $\mathrm{AD}$, the existing vitiligo lesions expanded and depigmentation progressed, which could be accounted for by the hypothesis that dupilumab affected the balance of the immune system towards polarizing $\mathrm{Th} / \mathrm{Tc} 1$ by blocking Th2. In vitiligo, both $\mathrm{CD} 4^{+}$and $\mathrm{CD} 8^{+} \mathrm{T}$ cells play an important role by producing interferon (IFN)- $\gamma$ and tumour necrosis factor- $\alpha(4,5)$, which are the signature cytokines of Th1/Tc1. Furthermore, Cheuk et al. (6) reported that, in skin from patients with vitiligo, where melanocytes are eradicated locally, $\mathrm{CD} 8^{+} \mathrm{CD} 49 \mathrm{a}^{+}$ resident memory $\mathrm{T}$ cells producing IFN- $\gamma$ accumulated in both the epidermis and dermis. In the current case, dupilumab suppressed Th2 cytokines, which may have activated existing $\mathrm{Th} / \mathrm{Tc} 1$ cells and $\mathrm{CD} 8^{+} \mathrm{CD} 49 \mathrm{a}^{+}$resi- dent memory $\mathrm{T}$ cells in small vitiligo lesions, resulting in enlargement of the skin patch affected by vitiligo vulgaris. Similarly, there have been several reports that dupilumab caused alopecia areata (7-9), which could be driven by $\mathrm{Th} / \mathrm{Tc} 1$ activation (10). As a possible mechanism, it was considered that dupilumab downregulates the Th2 pathway, thereby amplifying the Th1 pathway and promoting the development of alopecia areata (10). However, further research is needed to elucidate the mechanism of rapid progression of vitiligo vulgaris in patients who are receiving dupilumab.

\section{REFERENCES}

1. Simpson EL, Bieber T, Guttman-Yassky E, Beck LA, Blauvelt A, Cork MJ, et al. Two phase 3 trials of dupilumab versus placebo in atopic dermatitis. N Engl J Med 2016; 375: 2335-2348.

2. Uchida H, Kamata M, Mizukawa I, Watanabe A, Agematsu A, Nagata $M$, et al. Real-world effectiveness and safety of dupilumab for the treatment of atopic dermatitis in Japanese patients: a single-centre retrospective study. $\mathrm{Br}$ J Dermatol 2019; 181: 1083-1085.

3. Uchida H, Kamata M, Kato A, Mizukawa I, Watanabe A, Agematsu $A$, et al. One-year real-world clinical effectiveness, safety, and laboratory safety of dupilumab in Japanese adult patients with atopic dermatitis: a single-center retrospective study. J Am Acad Dermatol 2021; 84: 547-550.

4. Wańkowicz-Kalińska $A$, van den Wijngaard RM, Tigges $B J$, Westerhof W, Ogg GS, Cerundolo V, et al. Immunopolarization of CD4+ and CD8+ T cells to type-1-like is associated with melanocyte loss in human vitiligo. Lab Invest 2003; 83: 683-695.

5. Yang L, Wei Y, Sun Y, Shi W, Yang J, Zhu L, et al. Interferongamma inhibits melanogenesis and induces apoptosis in melanocytes: a pivotal role of CD8+ cytotoxic t lymphocytes in vitiligo. Acta Derm Venereol 2015; 95: 664-670.

6. Cheuk S, Schlums H, Gallais Sérézal I, Martini E, Chiang SC, Marquardt N, et al. CD49a expression defines tissue-resident CD8(+) T cells poised for cytotoxic function in human skin. Immunity 2017; 46: 287-300.

7. Kanda N, Koto M, Hoashi T, Saeki H. Case of alopecia areata during dupilumab treatment for atopic dermatitis. J Dermatol 2019; 46: e332-e333.

8. Maloney NJ, Worswick S, Cheng K. Development of alopecia in patients treated with dupilumab. Dermatol Ther 2019; 32: e12869.

9. Ständer S, Trense Y, Thaçi D, Ludwig RJ. Alopecia areata development in atopic dermatitis patients treated with dupiIumab. J Eur Acad Dermatol Venereol 2020; 34: e612-e613.

10. Ito T, Kageyama R, Nakazawa S, Honda T. Understanding the significance of cytokines and chemokines in the pathogenesis of alopecia areata. Exp Dermatol 2020; 29: 726-732. 